\title{
MARCO TEÓRICO PARA UN MODELO DE EQUILIBRIO GENERAL CON SECTOR HIDRÁULICO
}

\author{
Héctor Manuel Bravo Pérez* \\ Centro de Investigación y Docencia Económicas A. C. \\ Miguel Ángel Gutiérrez Andrade \\ Departamento de Sistemas, Universidad Autónoma Metropolitana-Azcapotzalco \\ (Recibido 7 de febrero 2003, aceptado 18 de julio 2003)

\section{Resumen} \\ En este trabajo se desarrolla un modelo de equilibrio general, estático y determinista, con \\ agentes representativos, para estudiar el manejo eficiente de los recursos hidráulicos. En \\ nuestro modelo, el capital, el trabajo y el agua representan los factores de la producción. \\ Finalmente, establecemos una estrategia de calibración para poder obtener recomendaciones \\ en materia de política fiscal, con el objetivo de hacer más eficiente el manejo del agua dentro \\ del proceso productivo, dependiendo de quien detente la propiedad del recurso: las unidades \\ familiares o el gobierno.
}

\begin{abstract}
In this paper, we develop a general equilibrium model, static, and deterministic with representative agents, useful to study the efficient management of water resources. In our model, capital, labor and water stand for the inputs in the production function. Finally, we state a calibration strategy to get a set of fiscal policy recommendations for the efficient management of water in the production process, depending on who holds the property of the resource: households or government.
\end{abstract}

Clasificación JEL: H3, D58, I3, Q25

Palabras clave: Política fiscal, Modelo de equilibrio general, Agua

* Carretera México-Toluca 3655. Lomas de Santa Fé, C. P. 01210, México D. F. Teléfono: +52(55)5727 9800 ext. 2333. Correo electrónico: hector.bravo@cide.edu 


\section{Introducción}

El artículo 27 de la Constitución Política de los Estados Unidos Mexicanos y su Ley Reglamentaria establecen que el agua es propiedad exclusiva de la nación. La nación tiene el derecho de transmitir el dominio del recurso a los particulares, constituyendo con esto la propiedad privada. Sin embargo, el gobierno mantiene por mandato constitucional la administración del recurso, instrumentando para ello políticas fiscales que garanticen su uso sustentable. Para que los agentes económicos (consumidores y empresas) puedan utilizar el agua en el proceso productivo, es necesario que el gobierno les otorgue una concesión, es decir, una cesión parcial de los derechos de propiedad. ${ }^{1}$

Las experiencias exitosas en la administración de recursos naturales cuando éstos se manejan en forma colectiva han sido numerosas. Al respecto, el trabajo clásico de Maass y Anderson (1986) analiza varios casos de administración del agua, en ámbitos sociales y económicos muy diversos, en los que la propiedad del agua es común. Sin embargo, no existe consenso sobre las formas de organización de las instituciones encargadas del manejo colectivo del recurso que aseguren un funcionamiento eficiente. En este sentido, Ostrom (1990) y (1992) hace una revisión de las distintas instituciones económicas diseñadas para el manejo común, entre particulares y gobierno, de los recursos naturales.

Hasta ahora no existe un estudio detallado de la eficiencia de las instituciones mexicanas encargadas de la administración del recurso, debido principalmente, a la heterogeneidad de la forma en que se obtiene el agua. Sin embargo, y a pesar de ser poco realista suponer que el gobierno mexicano abandone el control y el manejo del recurso, podemos plantear las siguientes preguntas:

1) ¿Cómo afectaría al bienestar económico, la aplicación de instrumentos de política fiscal (impuestos y/o subsidios) en el manejo del agua, en especial en situaciones de escasez relativa del recurso?

2) ¿Qué esquema de propiedad sobre el recurso es más conveniente para mejorar el bienestar económico?

3) ¿Qué esquema de propiedad hace más eficiente la política fiscal con el fin de preservar el recurso para las futuras generaciones?

Existen relativamente pocos trabajos sobre el análisis de las ventajas de un esquema de propiedad del agua sobre otro, es decir, propiedad pública o privada. Alguna evidencia empírica reciente que apoya la idea de propiedad privada se presenta en los trabajos de Aguilera-Klink (1992, 1994) y Weitzman (1974). Por otro lado, y aún cuando existen experiencias exitosas en el manejo de recursos naturales dentro de un esquema de propiedad pública, no han sido identificados los elementos económicos que aseguran la viabilidad de una forma de propiedad de los recursos con relación a otra.

En el caso del agua, pocos son los trabajos en los que se evalúa el desempeño de las instituciones dedicadas a su manejo. Entre los estudios más destacados encontramos el de Young et al.(1986), en el cual se desarrolla un modelo de

1 La indefinición en los derechos de propiedad del recurso puede generar ineficiencias, lo cual ha sido ampliamente discutido en la literatura. Véase, por ejemplo, Neher (1990). 
simulación para evaluar, en términos hidrológicos, las distintas alternativas. Asimismo, en el trabajo de Maass y Anderson (1986) se construye un modelo de simulación para cvaluar las instituciones encargadas del manejo del agua en distintos países.

En cste trabajo, construimos un modelo de equilibrio general, proponiendo un enfoque distinto a los utilizados en los trabajos mencionados. El documento se organiza de la siguiente manera. En la sección 2 se construye un modelo de equilibrio general para la evaluación de los instrumentos de política fiscal en el manejo eficiente del agua. En la sección 3 se introduce el comportamiento del gobierno. En la sección 4 se plantean las relaciones que definen el equilibrio y se propone una estrategia para la calibración del modelo. Finalmente, en la sección 5 se presentan las conclusiones, se establecen las limitaciones y ventajas del modelo, y se mencionan algunas líneas de investigación futura.

\section{El modelo propuesto}

En esta sección se construirá un modelo estático y determinista con agentes representativos en donde el capital, el trabajo y el agua en bruto son considerados como factores de la producción. El agua como uno de los insumos del proceso productivo se denominará agua de primer uso o agua útil. El agua puede ser ahorrada o consumida, esta decisión la puede tomar el agente consumidor o el gobierno, según quien detente la propiedad del recurso.

\subsection{Factores, bienes y precios}

El conjunto de factores de la producción en la economía se define como $F=\{$ trabajo, capital, agua superficial, agua subterránea\}. El conjunto de bienes que se producen en la economía se define como $G=\{$ bien del tipo 1 (emplea agua de primer uso, también llamada agua útil), bien del tipo 2 (emplea agua de primer uso y agua tratada), agua útil de origen superficial, agua útil de origen subterránco, agua residual y agua tratada\}. Se denotarán los factores, bienes y dotaciones de la siguiente manera:

$X_{1, j}=$ trabajo para producir el bien $j$,

$X_{k, j}=$ capital para producir el bien $j$,

$X_{2}=$ agua superficial consumida,

$X_{2, s}=$ agua superficial ahorrada,

$X_{3}=$ agua subterránea consumida,

$X_{3, s}=$ agua subterránea ahorrada,

$X_{4, j}=$ agua útil de origen superficial para producir el bien $j$,

$X_{5, j}=$ agua útil de origen subterráneo para producir el bien $j$,

$X_{6}=$ agua residual,

$X_{7}=$ agua tratada,

$X_{8}=$ bien de consumo del tipo 1 ,

$X_{9}=$ bien de consumo del tipo 2 ,

$W_{1}=$ cantidad de trabajo en la economía,

$W_{k}=$ cantidad de capital en la economía,

$W_{2}=$ cantidad inicial de agua superficial en la economía,

$W_{3}$ = cantidad inicial de agua subterránea en la economía. 
Los bienes pueden ser utilizados para consumo final o como insumos para producir otros bienes. Se supone que existe pleno empleo de todos los factores. El precio del bien (factor), $X_{i}\left(X_{i, j}\right)$, se denotará mediante $p_{i}\left(p_{i, j}\right)$.

Para que el agua pueda ser utilizada es necesario transformarla. El agua tal como se encuentra en sus fuentes originales no puede ser utilizada directamente en ningún proceso productivo y tiene que ser transformada en agua útil; este proceso puede ser tan complejo como la potabilización o tan simple como el transporte. Por otro lado, conjuntamente a la producción del bien de consumo 1 , se produce agua residual, que es la diferencia entre el agua útil empleada en el proceso productivo y el uso consuntivo, esto es, el volumen que se incorpora definitivamente al bien producido.

Vamos a suponer también que en esta economía existe una empresa que se encarga de limpiar el agua residual con una tecnología del tipo Leontief. Al ser tratada el agua residual recupera parcialmente las características de calidad originales. Este proceso es realizado por una empresa que utiliza al agua residual coino insumo, entre otros factores, y produce un bien denominado agua tratada, la que a su vez es un insumo de la tecnología utilizada en la producción del bien de consumo del tipo 2.

\subsection{Tecnología}

En esta economía existen cinco tipos de productores, cada uno de los cuales tiene un comportamiento optimizador y es precio aceptante. Se supone que las distintas tecnologías presentan rendimientos constantes a escala. Las demandas condicionadas o derivadas de los insumos para cada industria se obtienen como soluciones del problema de minimización de costos sujeto a la tecnología disponible.

A las demandas derivadas se les distinguirá con un asterisco y un doble subíndice $i, j$, donde $i$ denota el insumo y $j$ el bien al que será destinado el recurso. La cantidad total del bien producido se denotará mediante $Y_{j}$. Las funciones de producción que representan las tecnologías utilizadas se denotarán por $f_{j}$ y se supone que son homogéneas de grado uno en los factores, con lo cual se asegura que las demandas derivadas son funciones homogéneas de grado cero en los precios de los factores. A continuación se describen cada uno de los problemas de optimización que caracterizan el comportamiento de las distintas industrias y de los cuales se obtienen las demandas derivadas de factores.

\subsubsection{Producción del bien de consumo del tipo 1}

El bien de consumo del tipo 1 utiliza en su producción agua útil. El problema de minimización de costos sujeto a la tecnología para la producción del bien del tipo 1 está dado por:

$$
\begin{array}{cc}
\text { Minimizar } & p_{1} X_{1,8}+p_{k} X_{k, 8}+p_{4} X_{4,8}+p_{5} X_{5,8}+\tau_{6} m\left(X_{4,8}+X_{5,8}\right), \\
& \text { sujeto a : } Y_{8}=f_{8}\left(X_{1,8}, X_{k, 8}, X_{4,8}, X_{5,8}\right) .
\end{array}
$$

Una parte del agua extraída es consumida y otra descargada a un cuerpo receptor. En esta economía, el agua descargada se considera como un bien intermedio 
que sirve como insumo al productor de agua tratada. Nótese que el productor del bien de consumo 1 paga por el uso del agua, como un insumo, más un gravamen por la cantidad de agua destruida.

De la solución del problema de optimización anterior se obtienen las demandas de factores, $\left(X_{1,8}^{*}, X_{k, 8}^{*}, X_{4,8}^{*}, X_{5,8}^{*}\right)$, las cuales son funciones de los siguientes parámetros y cantidades: $\left(p_{1}, p_{k}, p_{4}, p_{5}, \tau_{6}, m, Y_{8}\right)$, donde $\tau_{6}$ es el impuesto pagado por la destrucción de agua y $m$ es el porcentaje de uso consuntivo, por lo que el agua que queda libre para otros usos es: $X_{6}^{*}=(1-m)\left(X_{4,8}^{*}+X_{5,8}^{*}\right)$.

\subsubsection{Producción de agua tratada}

La tecnología para producir agua tratada restituye en el agua, al menos parcialmente, la calidad necesaria para hacerla útil nuevamente. El productor de agua tratada, que utiliza el agua residual como insumo, resuelve el siguiente problema de optimización:

$$
\begin{array}{ll}
\text { Minimizar } & p_{1} X_{1,7}+p_{k} X_{k, 7}+p_{6} X_{6,7}, \\
\text { sujeto a : } & Y_{7}=f_{7}\left(X_{1,7}, X_{k, 7}, X_{6,7}\right) .
\end{array}
$$

Las demandas condicionales son denotadas por: $\left(X_{1,7}^{*}, X_{k, 7}^{*}, X_{6,7}^{*}\right)$, las cuales están en función de los siguientes precios y cantidades: $\left(p_{1}, p_{k}, p_{6}, Y_{7}\right)$.

\subsubsection{Producción del bien de consumo del tipo 2}

El otro tipo de bien de consumo en la economía puede producirse con ambos tipos de agua: tratada y de primer uso sin generar desperdicio. Ejemplos de este tipo de bienes pueden ser el regado de calles, algunos procesos de enfriamiento o el riego de algunos cultivos que no requieran agua de cierta calidad. Por lo tanto, si se emplea agua de primer uso, ésta se gravará, mientras que si se emplea agua tratada se paga el precio del mercado. El problema de decisión que resuelve el productor del bien de consumo 2 es:

$$
\begin{array}{cl}
\text { Minimizar } & p_{1} X_{1,9}+p_{k} X_{k, 9}+p_{4}\left(1+\tau_{4}\right) X_{4,9}+p_{5}\left(1+\tau_{5}\right) X_{5,9}+p_{7} X_{7,9}, \\
& \text { sujeto a: } Y_{9}=f_{9}\left(X_{1,9}, X_{k, 9}, X_{4,9}, X_{5,9}, X_{7,9}\right) .
\end{array}
$$

Las demandas condicionales están dadas por: $\left(X_{1,9}^{*}, X_{k, 9}^{*}, X_{4,9}^{*}, X_{5,9}^{*}, X_{7,9}^{*}\right)$, las cuales están en función de $\left(p_{1}, p_{k}, p_{\mathbf{4}}, \tau_{4}, p_{5}, \tau_{5}, p_{7}, Y_{9}\right)$.

Por otro lado, como ya ha sido mencionado, en esta economía el agua no puede ser utilizada sin un proceso previo que transforma el agua en bruto en agua útil y se distingue por su procedencia: superficial o subterránea. Supondremos que los agentes que se encargan de producir el agua útil, se comportan como si resolvieran los dos problemas que a continuación se describen.

\subsubsection{Producción de agua útil de fuentes superficiales}

El problema de minimización de costos para la producción de agua útil de fuentes superficiales está dado por:

$$
\text { Minimizar } p_{1} X_{1,4}+p_{k} X_{k, 4}+p_{2}\left(1+\tau_{2}\right) X_{2,4},
$$




$$
\text { sujeto a : } Y_{4}=f_{4}\left(X_{1,4}, X_{k, 4}, X_{2,4}\right) \text {. }
$$

Las demandas condicionales están dadas por: $\left(X_{1,4}^{*}, X_{k, 4}^{*}, X_{2,4}^{*}\right)$, las cuales están en función de $\left(p_{1}, p_{k}, p_{2}, \tau_{2}, Y_{4}\right)$, donde $\tau_{2}$ es el impuesto que se debe pagar por la extracción de agua superficial.

\subsubsection{Producción de agua útil de fuentes subterráneas}

Para la producción de agua útil proveniente de fuentes subterráneas el problema de minimización es:

$$
\begin{gathered}
\text { Minimizar } p_{1} X_{1,5}+p_{k} X_{k, 5}+p_{3}\left(1+\tau_{3}\right) X_{3,5}, \\
\text { sujeto a : } Y_{5}=f_{5}\left(X_{1,5}, X_{k, 5}, X_{3,5}\right) .
\end{gathered}
$$

Las demandas condicionales están dadas por: $\left(X_{1,5}^{*}, X_{k, 5}^{*}, X_{3,5}^{*}\right)$, las cuales están en función de $\left(p_{1}, p_{k}, p_{3}, \tau_{3}, Y_{5}\right)$, donde $\tau_{3}$ es el impuesto que se debe pagar por la extracción de agua subterránea.

\subsection{Consumidores representativos}

En esta sección, analizaremos dos tipos distintos de Derechos de Propiedad (DDP) del agua: propiedad pública, en la cual los recursos hidráulicos de la economía pertenecen al gobierno, y propiedad privada, cuando el agua pertenece a los agentes privados, en ambos casos el agua se oferta inelásticamente. Estos dos tipos de propiedad afectan tanto a las restricciones presupuestales de los consumidores como a los beneficios de las empresas productoras de agua útil y, por ende, a los niveles de bienestar que se alcanzan en la economía. Para realizar la evaluación de cada una de las formas de propiedad, caracterizaremos los beneficios y las restricciones presupuestales en cada caso.

Es importante recordar que cuando el gobierno es propietario del recurso, los ingresos derivados de la venta de agua sólo pueden destinarse a la conservación del recurso, esto es, no es posible transferir a los consumidores las rentas derivadas de la venta del agua, esta restricción se impone con la idea de incorporar la política seguida en México, denominada "lo del agua al agua". Por lo tanto, los beneficios que obtienen los productores de agua útil, cuando la propiedad del agua recae en ellos, es la siguiente:

$$
\Pi_{i, j}=p_{j} Y_{j}-p_{1} X_{1, j}-p_{k} X_{k, j}-p_{i}\left(1+\tau_{i}\right) X_{i, j},
$$

$\operatorname{con} j=4,5, i=2,3$ y $\tau_{i}$ es el impuesto a la extracción. La parte de la demanda en esta economía está representada por dos tipos diferentes de agentes, $H_{1}$ y $\mathrm{H}_{2}$, cuyas preferencias son representadas por medio de funciones de utilidad estrictamente cuasi-cóncavas y dos veces diferenciables.

Los consumidores derivan utilidad de dos tipos de bienes, el bien de consumo 1 que utiliza en su producción agua de primer uso y el bien de consumo 2 que utiliza tanto agua de primer uso como agua tratada. El consumidor dueño del agua toma la decisión de intercambiar el agua o de ahorrarla. Se supone que las dotaciones iniciales de los factores que cada uno de los agentes posee son distintas. El agente $H_{1}$ posee todo el capital que hay en la economía y participa en el mercado del trabajo, mientras que el agente $H_{2}$ posee toda el agua 
y también participa en el mercado laboral. Los consumidores son racionales y resuelven los siguientes problemas de maximización de utilidad sujetos a su restricción presupuestal.

\subsubsection{Problema de decisión del agente $H_{1}$}

El problema de maximización de utilidad que resuelve el consumidor $H_{1}$ está dado por:

$$
\begin{gathered}
\text { Maximizar } U_{H_{1}}\left(X_{8, H_{1}}, X_{9, H_{1}}\right), \\
\text { sujeto a : } p_{8} X_{8, H_{1}}+p_{9} X_{9, H_{1}} \leq p_{1} W_{1, H_{1}}+p_{k} W_{k}+T_{H_{1}} \equiv I D_{H_{1}},
\end{gathered}
$$

donde $T_{H_{1}}$ son las transferencias gubernamentales. Como es habitual, podemos obtener las demandas Marshallianas al resolver el problema anterior, de donde se siguen las relaciones:

$$
X_{8, H_{1}}^{*}=X_{8, H_{1}}^{*}\left(p_{8}, p_{9} X_{9, H_{1}}, I D_{H_{1}}\right)
$$

y

$$
X_{9, H_{1}}^{*}=X_{9, H_{1}}^{*}\left(p_{9}, p_{8} X_{8, H_{1}}, I D_{H_{1}}\right)
$$

\subsubsection{Problema de decisión del agente $H_{2}$}

El agente $\mathrm{H}_{2}$ es propietario del agua y tiene que decidir cuanta agua ahorrar. En este caso, el problema de maximización de utilidad que resuelve el consumidor $H_{2}$ está dado por:

$$
\begin{gathered}
\text { Maximizar } U_{H_{2}}\left(X_{8, H_{2}}, X_{9, H_{2}}, X_{2, s}, X_{3, s}\right), \\
\text { sujeto a : } p_{8} X_{8, H_{2}}+p_{9} X_{9, H_{2}}+p_{2, s} X_{2, s}+p_{3, s} X_{3, s} \leq I D_{H_{2}} .
\end{gathered}
$$

El valor de $I D_{H_{2}}$ queda definido de la siguiente manera:

$$
I D_{H_{2}}=p_{1} W_{1, H_{2}}+p_{2}\left(1-\mu_{2}\right) W_{2}+p_{3}\left(1-\mu_{3}\right) W_{3}+T_{H_{2}}+\Pi_{4}+\Pi_{5} \text {. }
$$

En el caso de propiedad privada $\mu_{2}=\mu_{3}=0 \mathrm{y}$ en el caso de propiedad pública $\mu_{2}=\mu_{3}=1$. Aquí, $T_{H_{2}}$ representa las transferencias gubernamentales a este agente y $\Pi_{4}$ y $\Pi_{5}$ son los brueficios que el agente $H_{2}$ obtiene de la producción de agua útil, tanto superficial como subterránea. Al igual que en el caso anterior, podemos obtener las demandas Marshallianas, mismas que denotamos por $\left(X_{8, H_{2}}^{*}, X_{9, H_{2}}^{*}, X_{2, s}^{*}, X_{3, s}^{*}\right)$ y que dependen de $\left(p_{8}, p_{9}, p_{2, s}, p_{3, s}, I D_{H_{2}}\right)$.

\section{Comportamiento del gobierno}

El gobierno pue le ser poseedor o no de los recursos hidráulicos de la economía, en cualquier caso gr rva los siguientes conceptos: la destrucción de agua, la producción de i , uit residual, el consumo, el capital y el trabain. Además, el gobierno transfiere en forma directa a los consumidores el total $c$ su ingreso por impuestos, pero no así los de la venta de agua, que como ha sido mencionado antes, los utiliza para mantener los niveles de agua ahorrada. Por lo tanto, en 
esta economía el gobierno sólo recauda impuestos y redistribuye el ingreso. Así, la restricción presupuestal del gobierno es la siguiente:

$$
R^{c}+R^{l}+R^{k}+\tau_{6} X_{6}^{*}+p_{2}\left(1-\mu_{2}\right) X_{2}^{*}+p_{3}\left(1-\mu_{3}\right) X_{3}^{*}=Q,
$$

donde

$$
Q=p_{2, s}\left(1-\mu_{2}\right) X_{2, s}^{*}+p_{3, s}\left(1-\mu_{3}\right) X_{3, s}^{*}+T_{H_{1}}+T_{H_{2}} .
$$

Como antes, en el caso de propiedad privada $\mu_{2}=\mu_{3}=0 \mathrm{y}$ en el caso de propiedad pública $\mu_{2}=\mu_{3}=1$. Finalmente, las recaudaciones por consumo, trabajo y capital son respectivamente:

$$
\begin{gathered}
R^{c}=\sum_{H_{j}} \sum_{i=8}^{9} \tau_{i}^{c} p_{i} X_{i, H_{j}}^{*}, \\
R^{l}=p_{1} \sum_{i=8}^{9} \tau_{i}^{l} X_{1, i}^{*}
\end{gathered}
$$

y

$$
R^{k}=p_{k} \sum_{i=8}^{9} \tau_{i}^{k} X_{k, i}^{*} .
$$

\section{Determinación del equilibrio}

Un cquilibrio para esta economía se define como: un vector de precios de bienes y factores, un vector de planes de consumo y de producción, y un nivel de recaudación tales que satisfagan las siguientes condiciones. En los mercados de bienes, la cantidad producida del bien final es igual a la suma de la demanda intermedia del bien más la demanda final del bien, esto es:

$$
Y_{j}^{*}=\sum_{i} X_{i, j}^{*}+\sum_{h=H_{1}, H_{2}} X_{j, h}^{*}, \quad \forall j .
$$

Las condiciones de equilibrio que deben satisfacer los mercados de factores de la producción son las siguientes. En particular, en el mercado de trabajo se debe cumplir que:

$$
W_{1}=\sum_{j} X_{1, j}^{*}
$$

Asimismo, en el mercado de capital se debe satisfacer:

$$
W_{k}=\sum_{j} X_{k, j}^{*}
$$

La condición que se debe cumplir en el mercado de agua superficial está dada por:

$$
W_{2}=\sum_{j} X_{2, j}^{*}+X_{2, s}^{*}
$$


Finalmente, en el mercado de agua subterránea se debe cumplir que:

$$
W_{3}=\sum_{j} X_{3, j}^{*}+X_{3, s}^{*} .
$$

Con el propósito de cerrar el modelo, se incorpora en la estructura de la economía el comportamiento del sector público. En este caso, se supone que el nivel de recaudación es igual a los pagos fiscales realizados por los agentes económicos.

\subsection{Información requerida}

El supuesto de existencia de un equilibrio nos obliga a construir un esquema de contabilidad que asegure que se cumplan todas las condiciones antes mencionadas. Se identifican cuatro conjuntos de condiciones de equilibrio que deben satisfacerse: a) en todos los mercados de bienes la demanda es igual a la oferta; b) no se alcanzan beneficios positivos en ninguna industria; c) todos los agentes satisfacen sus demandas cumpliendo con su restricción presupuestal y d) el gobierno no incurre en déficit.

Para construir la base de datos, que requiere el equilibrio computable, se utiliza información de diversas fuentes, que bien podrían ser inconsistentes. Por lo tanto, el primer paso es hacer consistente la base de datos para el año de referencia que se haya elegido. Las matrices SAM (Social Accounting Matrices) poseen una estructura adecuada para revisar la consistencia de la información, ya que contienen para cada agente del modelo el ingreso y el gasto, así como las ventas y compras de insumos para cada bien. Véase, por ejemplo, Ginsburgh y Keyzer (1997).

La información que sería útil para una posible calibración del modelo podría ser obtenida de las siguientes fuentes: Sistema de Cuentas Nacionales de México, Encuesta Ingreso Gasto, Censos Económicos, Sistema de Información Municipal Básica y Matriz de Insumo-Producto del Instituto Nacional de Estadística Geografía e Informática. Con relación a los parámetros técnicos de la utilización del agua la fuente sería el Instituto Mexicano de Tecnología del Agua.

\subsection{Calibración del modelo}

Una vez que la información haya sido recopilada y sistematizada, y que la metodología a partir de la cual se evaluarán los distintos DDP del agua se hayan establecido, se tendría que proceder con la determinación del equilibrio. Para resolver este tipo de problemas, primero, habría que encontrar los parámetros que reproducen el equilibrio de referencia ("benchmark equilibrium"), proceso que es conocido como calibración. Una vez determinados estos parámetros es posible realizar ejercicios de estática comparativa, es decir, encontrar nuevas soluciones de equilibrio ante modificaciones de algunas variables exógenas ("counterfactual equilibrium").

Para la solución de este problema podría considerarse, en primera instancia, la metodología sugerida por Shoven (1995), Scarf (1973) y Ginsburgh y Keyzer (1997). El algoritmo propuesto por estos autores para determinar un equilibrio computable puede resumirse en los siguientes pasos: 
1) Se postula una conjetura inicial sobre los precios de los factores, los bienes demandados y sobre la recaudación.

2) Se calculan los precios de los bienes, recordando que son iguales a sus costos marginales.

3) Se calcula el valor de la dotación inicial de los recursos para cada agente $h$ y con ella la demanda de cada uno de los agentes por los bienes que se encuentran en su canasta.

4) Las cantidades producidas de bienes deben ser iguales a las demandas finales.

5) Se determinan las demandas derivadas de factores a partir de los precios y de las cantidades producidas.

6) Se comparan las demandas derivadas de factores con la dotación de factores en la economía y la recaudación calculada con la observada en el equilibrio. Si se cumplen estas dos igualdades, se ha encontrado entonces un equilibrio. De no ser así, se repiten los pasos anteriores a partir del número 2), tomando los últimos valores calculados como los valores conjeturados.

Para calibrar el modelo es necesario especificar formas funcionales de las tecnologías y preferencias, a partir de las cuales se definirán los parámetros por estimar. A continuación, se definen las relaciones funcionales utilizadas en este trabajo. Es importante proponer una especificación funcional que, por un lado, reproduzca el equilibrio observado $y$, por otro lado, explique el comportamiento de los agentes y los mercados ante cambios en las variables exógenas.

Como es habitual en este tipo de modelos, se utilizarán funciones de producción del tipo Leontief en el que aparecen como parámetros las cantidades de bienes intermedios utilizados como insumos. Esta función de producción pertenece a la familia CES (Constant Elasticity of Substitution). Específicamente, las funciones utilizadas son las siguientes:

$$
\text { Bien } j: \quad Y_{j}=\operatorname{Min}\left\{\frac{X_{i, j}}{a_{i, j}}, \frac{V_{j}}{\nu_{j}}\right\}, \quad \forall j=4,5,7,8,9 \text {, }
$$

donde $a_{i j}$ son los requerimientos unitarios del factor $i$ para producir el bien $j$, $V_{j}$ es la cantidad de valor añadido necesario para producir una unidad del bien $j$ y $\nu_{j}$ es el requerimiento unitario de valor añadido para producir una unidad del bien $j$. Las funciones que definen los valores añadidos en cada una de las funciones de producción son también de elasticidad constante:

$$
V_{j}=\frac{1}{\varphi_{j}}\left(\sum_{i} \beta_{i, j} X_{i, j}^{-\rho_{j}}\right)^{-\frac{1}{\rho_{j}}}, \quad \forall j=1,2,4, \ldots, 8,
$$

donde $\rho_{j}$ es el parámetro de sustitución, $\varphi_{j}$ es un coeficiente tecnológico y $\beta_{i, j}$ es un parámetro de requerimientos. Por lo tanto, la demanda final estará determinada por la demanda de los dos de consumidores, los cuales tienen una función de utilidad del tipo Cobb-Douglas, de tal forma que:

$$
\text { Agente } H_{1}: \quad U_{H_{1}}=X_{8, H_{1}}^{\alpha_{H_{1}}} X_{9, H_{1}}^{1-\alpha_{H_{1}}}
$$




$$
\begin{gathered}
\text { Agente } H_{2}: U_{H_{2}}=X_{8, H_{2}}^{\alpha_{1, H_{2}}} X_{9, H_{2}}^{\alpha_{2, H}} X_{2 s}^{\alpha_{3, H_{2}}} X_{3 s}^{\alpha_{4, H_{2}}}, \\
\operatorname{con} \sum_{i=1}^{4} \alpha_{i, H_{2}}=1,
\end{gathered}
$$

donde los valores de $\alpha_{i, j}$ representan la proporción del ingreso que el consumidor $H_{2}$ gasta en el bien $i$.

La calibración es un proceso determinista por medio del cual es posible estimar valores numéricos de los distintos parámetros que intervienen en el modelo: los relacionados con las distintas tecnologías, los de preferencias de los distintos agentes y los de naturaleza fiscal. En otras palabras, la calibración consiste en estimar aquellos parámetros que permitan reproducir el equilibrio de referencia que se describe en la matriz de contabilidad social. Esta asignación no es arbitraria, sino que obedece al comportamiento optimizador de los agentes. A excepción de los parámetros relacionados con la elasticidad de sustitución en la función de producción, todos los demás parámetros son determinados por la información que proporciona la matriz de contabilidad social.

\section{Conclusiones}

En México, los elementos que definen los DDP sobre el agua están delineados en la Ley de Aguas Nacionales (LAN). Este es el marco regulatorio que el gobierno mexicano ha creado con el fin de que los agentes económicos puedan hacer uso del agua, recurso que se considera propiedad de la nación.

Bajo el supuesto de que el estado mantenga la obligación de administrar los recursos hidráulicos del país, aún en el hipotético caso en que la propiedad del agua fuera privada, se plantea la aplicación de instrumentos de política fiscal para el manejo del recurso. Con este fin se construyó un modelo que permitiera simular y evaluar diferentes alternativas de propiedad y sus efectos en el bienestar. La base de datos necesaria para el funcionamiento del modelo se denomina Matriz de Contabilidad Social (SAM), en ella se reflejan todos los elementos esenciales del equilibrio de referencia, a partir del cual se calibra el modelo. Una característica importante que debió incorporarse a la matriz, y que se hizo sólo parcialmente, es el efecto de las externalidades negativas. Por otra parte, dada la información disponible para el caso mexicano, se incorporaron los ajustes indispensables para tomar en cuenta los elementos de la producción de agua residual. Por lo tanto, las conclusiones a las que llegamos, con relación a las hipótesis originales de este trabajo, son:

(i) Si se aplica un impuesto al uso consuntivo, los niveles de bienestar no se ven afectados en condiciones de propiedad y de abundancia relativa diferentes, salvo en el caso de que exista abundancia y propiedad privada.

(ii) Si se aplica un subsidio a la producción de agua residual, tanto la propiedad como la abundancia relativa tienen efectos en el bier 'star. Cuando la propiedad del agua es privada, el subsidio tiene efectos contrarios según la abundancia relativa del recurso, mientras que cuando la propiedad es pública el efecto es positivo con un mayor impacto en el caso de zonas de abundancia relativa. 
Con relación al efecto en la conservación del agua, los resultados son:

(i) Gravar el uso consuntivo es efectivo cuando la propiedad del agua es privada, sin importar la abundancia o escasez relativa, y neutral cuando el agua es de propiedad pública.

(ii) Subsidiar la producción de agua residual es negativo cuando la propiedad es privada, independientemente de la abundancia relativa del recurso, y es una mala política de conservación con propiedad pública. El peor de los casos ocurre cuando esta política se aplica a zonas de escasez relativa.

Al resumir los resultados anteriores, podemos decir que en términos de bienestar social, subsidiar la producción de agua residual cuando la propiedad del agua es pública resulta ser la mejor política, mientras que gravar el uso consuntivo es neutral. Para el caso de propiedad privada los resultados son ambiguos dependiendo de la abundancia del recurso. Ahora bien con respecto a la conservación, la mejor política se alcanza cuando tenemos propiedad privada y se grava el uso consuntivo, independientemente de la abundancia o escasez relativa del agua. Subsidiar el agua residual con propiedad privada puede llevar a la destrucción del recurso.

Con el uso del modelo construido en este trabajo, es posible evaluar los distintos instrumentos de política fiscal y las alternativas de propiedad del agua. Sin embargo, es necesario reconocer que algunos de sus elementos pueden ser mejorados: 1) la oferta de agua en bruto se supuso, como es común en estos modelos, infinítamente inelástica; 2) los diferentes tipos de propiedad podrían tener efectos por el lado de la oferta y 3 ) finalmente, es un hecho irrebatible, que el proceso de descentralización que se ha observado en México en los últimos años de los distritos de riego y, en general, del sector hidráulico, a incentivado al cambio tecnológico. Por lo tanto, suponer que la tecnología en el uso del agua. es constante, es un supuesto que debe relajarse con el fin de obtener resultados más realistas.

\section{Bibliografía}

Aguilera-Klink, F. (1992). El Fin de la Tragedia de los Comunes. Ecología Política, enero, pp. $137-145$.

Aguilera-Klink, F. (1994). Pigou and Coase Reconsidered. Land Economics, 70(3), pp. 386-390.

Ginsburgh, V., and M. Keyzer (1997). The Structure of Applied General Equilibrium Models. The MIT Press, Cambridge Massachusetts.

Maass, A., and R. Anderson (1986), ... and the Desert Shall Rejoice. Conflict, Growth, and Justice in Arid Environments. Robert E. Krieger Publishing Company, Inc. Malabar, Florida.

Neher, P. (1990). Natural Resource Economics, Conservation and Explotation. Cambridge University Press, Cambridge.

Ostrom, E. (1990). Governing the Commons. The Evolution of Institutions for Collective Action. Cambridge University Press.

Ostrom, E. (1992). Crafting Institutions for Self-Governing Irrigation Systems. Institute for Contemporary Studies.

Scarf, H. (1973). The Computation of Economic Equilibria. Yale University Press, New Haven.

Shoven, T. (1995). Applying General Equilibrium. Cambridge University Press, Cambridge. 
Weitzman, M. (1974). Free Access vs Private Ownership as Alternative Systems for Managing Common Property. Journal of Economic Theory, 8, pp. 225-234.

Young, R., J. Daubert, and H. Morel-Seytoux (1986). Evaluating Institutional Alternatives for Managing an Interrelated Stream-Aquifer System. American Journal of Agricultural Economics, November, pp. 787-797. 